\title{
Quiste cerebral como complicación tardía inusual de lobectomía temporal en epilepsia farmacorresistente: reporte de un caso.
}

Brain cyst as an unusual delayed complication of temporal lobectomy in drug-resistant epilepsy: a case report.

\author{
Elliot Barreto-Acevedo ${ }^{1,}$, Alicia Becerra-Zegarra 2, b , Mirla Villafuerte-Espinoza ${ }^{1, a}$
}

\section{RESUMEN}

La lobectomía temporal anterior es una técnica que ha probado, con un muy alto nivel de evidencia (60-73\% de los casos), alcanzar un estado libre de convulsiones en pacientes con epilepsia focal farmacorresistente del lóbulo temporal. Se trata de una técnica que aun cuando ha demostrado ser segura, no está del todo libre de complicaciones. Se han reportado, por ejemplo, cuadrantanopsia homónima superior y depresión como las complicaciones más frecuentes, en tanto que la ocurrencia de un quiste cerebral sintomático de instauración tardía es muy poco usual. Se describe el caso de una paciente sometida a lobectomía temporal derecha que presentó esta infrecuente complicación, y se incluyen una pertinente revisión de la literatura y mecanismos fisiopatogénicos propuestos.

PALABRAS CLAVE: Quiste, complicación tardía, cirugía de epilepsia, lobectomía temporal, epilepsia del lóbulo temporal.

\section{SUMMARY}

Anterior temporal lobectomy is a technique that has proven, with a very high level of evidence $(60-73 \%$ of the cases), to reach a seizure-free status in patients with drug-resistant focal temporal lobe epilepsy. It is a technique that although generally safe, cannot be considered entirely free of complications. Superior homonymus quadrantanopsia and depression have been reported, for instance, as the most frequent complications. While the occurrence of a lateonset symptomatic brain cyst, is very rare. The case of a patient who was subjected to right temporal lobectomy and presented this unusual complication is described here, with inclusion of a review ofpertinent literature and proposed pathophysiological mechanisms.

KEYWORDS: Cyst, delayed complication, epilepsy surgery, temporal lobectomy, temporal lobe epilepsy.

1 Unidad de Epilepsia, Departamento de Neurología, Hospital Nacional Edgardo Rebagliati Martins, EsSalud. Lima, Perú.

2 Servicio de Neurocirugía Funcional, Hospital Nacional Edgardo Rebagliati Martins, EsSalud. Lima, Perú.

a Médico-Neurólogo; ${ }^{\text {b }}$ Médico-Neurocirujano de Epilepsia. 
Quiste cerebral como complicación tardía inusual de lobectomía temporal en epilepsia farmacorresistente.

\section{INTRODUCCIÓN}

La cirugía resectiva de epilepsia es el tratamiento de elección para muchos tipos de epilepsia focal farmacorresistente $(1,2)$. La lobectomía temporal anterior es la técnica que ha probado, con nivel de evidencia IA, obtener libertad de crisis entre un $\sim 60$ a $73 \%$ de los pacientes con epilepsia focal farmacorresistente del lóbulo temporal $(3,4)$. Es una técnica que además ha demostrado ser segura, aunque no libre de complicaciones. En la literatura se han reportado la cuadrantanopsia homónima superior y los trastornos psiquiátricos, en especial la depresión y ansiedad, como las complicaciones más frecuentes (5). Es muy infrecuente la presentación de un quiste cerebral sintomático de instauración tardía como complicación de esta técnica. Describimos el caso de una paciente quien presentó esta inusual complicación y revisamos la literatura al respecto.

\section{Descripción del caso}

Mujer de 52 años, diestra, asistente administrativa en empresa de transporte, sin factores de riesgo para epilepsia durante sus primeros años de vida, desarrollo psicomotor normal y escolaridad adecuada. Tiene antecedentes de secuela de polio (paresia crural izquierda), quemadura en miembro superior izquierdo y fractura de fémur izquierdo. Estos dos últimos eventos estuvieron asociados a crisis epilépticas; tuvo que ser intervenida de colocación de tornillos con placa metálica en el fémur izquierdo (figura 1A). Deambula con muletas y realiza sus actividades de la vida diaria. Tiene epilepsia focal farmacorresistente, con edad de inicio a los 22 años. Presentaba crisis focales con alteración de conciencia: aura de miedo y "sensación de no ser ella", seguidos de parada comportamental con pérdida del contacto con el entorno, asociada con automatismos orodeglutorios y manuales bilaterales a predominio derecho. En algunas crisis, los testigos han evidenciado habla periictal. Las crisis eran diurnas en vigilia y la frecuencia era de 1 a 2 /semana; ha presentado ocasionalmente crisis tónico - clónicas bilaterales. Recibió Valproato y Lamotrigina en monoterapia y en combinación, a dosis óptimas sin éxito. Esquema actual: Levetiracetam $3 \mathrm{~g}$ /día y Carbamazepina $600 \mathrm{mg} /$ día. Fue estudiada en La Unidad de Epilepsia del Hospital Rebagliati con estudios prequirúrgicos en fase I: El videoEEG de 78 horas con retiro completo de medicación anticrisis, evidenció descargas epileptiformes interictales en región temporal anterior derecha y se capturaron 4 crisis focales habituales, cuya actividad eléctrica ictal y semiología determinaron zona de inicio ictal en región temporal mesial derecha (figura 2). La tomografía cerebral no evidenció anormalidades; no se pudo realizar resonancia de encéfalo debido a que era portadora de dispositivo metálico en el cuerpo. Se le realizó PET scan cerebral interictal que evidenció hipometabolismo temporal derecho (figura 1 (B y C)). La evaluación neuropsicológica evidenció moderado compromiso de la memoria no verbal y preservación de la memoria verbal, con dominancia izquierda para el lenguaje, sin otros hallazgos de relevancia. En junta médica multidisciplinar de La Unidad de Epilepsia, se concluyó que la paciente era candidata de lobectomía temporal derecha, la paciente y la familia autorizaron la cirugía.

La paciente fue intervenida de craneotomía y lobectomía temporal anterior derecha: se resecó 5,5 $\mathrm{cm}$ de neocórtex lateral desde el polo y se realizó amigdalohipocampectomía. La tomografía de control postoperatorio a las 48 horas solo evidenció el área de resección (figura 3(A y B)). No hubo complicaciones postquirúrgicas tempranas. El estudio anatomopatológico evidenció esclerosis hipocampal. Respecto al control de crisis, en los siguiente 16 meses la paciente evolucionó en Engel Ib (libertad de crisis incapacitantes que cursaran con alteración de conciencia) y solo presentó auras aisladas de miedo, de pocos segundos 1 vez cada 1 a 2 meses.

A los 7 meses de la lobectomía temporal anterior derecha, se le realizó una tomografía cerebral debido a que la paciente refería leve e intermitente cefalea en la zona operatoria y se evidenció, además de la zona de resección un pequeño quiste subcortical en el límite de la ínsula posterior y la región posterior del lóbulo temporal derecho (figura 3(C)). Se le recomendó observar la evolución de la sintomatología y un control tomográfico en 6 meses o antes si hubieses deterioro neurológico. En los meses siguientes, debido a la instauración de la pandemia por COVID-19, la paciente no tuvo más controles ambulatorios y no se realizó la tomografía de control. Luego de 17 meses de la lobectomía temporal anterior derecha, habiendo mantenido su medicación anticrisis en las mismas dosis, presentó hemiparesia izquierda moderada de instauración en 3 semanas, exacerbación de la cefalea y recurrencia de 2 crisis focales con alteración de conciencia, por ello fue nuevamente hospitalizada. El examen físico evidenció a una paciente lúcida, orientada, con hemiparesia izquierda directa (fuerza muscular $3+/ 5$ ) y piramidalismo izquierdo, sin otros hallazgos clínicos contributorios. En la tomografía 


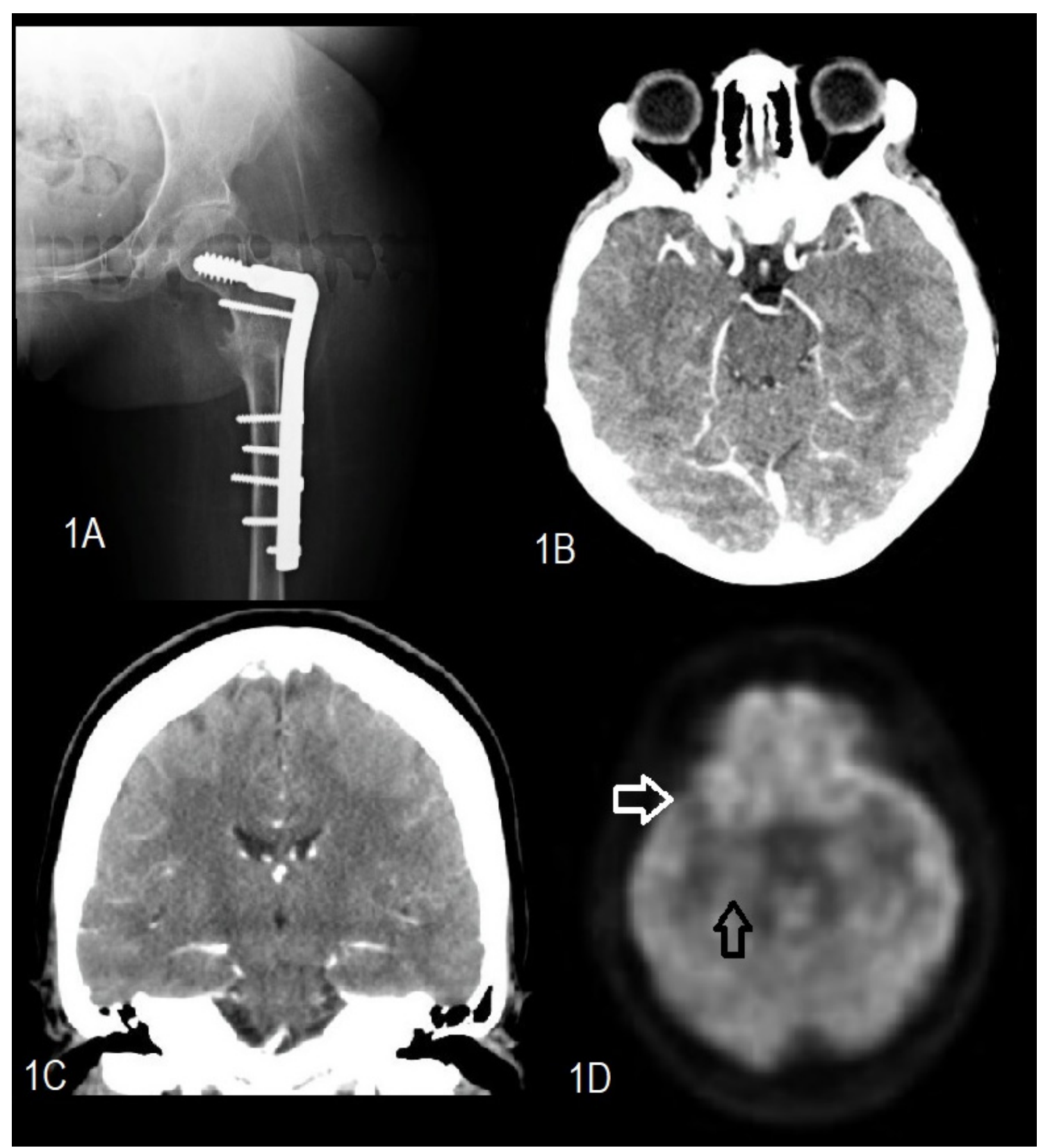

Figura 1. Radiografía que evidencia fractura de femur izquierdo fijada con tornillos y placa metálica (1A). Tomografía cerebral con contraste como estudio pre-cirugía de epilepsia, no evidencia lesiones (1B y 1C). PET scan cerebral interictal, las flechas señalan hipometabolismo temporal derecho (1D).

de cerebro se evidenció un incremento significativo del volumen de la lesión quística intracerebral, con extensión hacia la región posterior del lóbulo temporal derecho e ínsula derecha; y generaba efecto de masa con desviación de la línea media y colapso del ventrículo lateral derecho (figura 3 (D, E y F)). El EEG de 30 minutos evidenció ondas delta polimorfas, de mediano voltaje en derivaciones fronto-temporales derechas, sin evidenciar actividad epileptiforme. La paciente fue intervenida quirúrgicamente de una nueva craneotomía, se evidenció colección de líquido cefalorraquídeo a moderada presión. No se realizó exploración endoscópica en el interior del quiste que permitiera identificar la estructura que genere el fenómeno de válvula. Se realizó drenaje del quiste mediante derivación cistoperitoneal. La tomografía cerebral posterior al drenaje evidenció una reducción significativa del volumen del quiste (figura $3(\mathrm{G} \mathrm{y} \mathrm{H})$ ). Luego de 4 semanas de la colocación del drenaje, la paciente no ha vuelto a presentar crisis epilépticas, la 
Quiste cerebral como complicación tardía inusual de lobectomía temporal en epilepsia farmacorresistente.

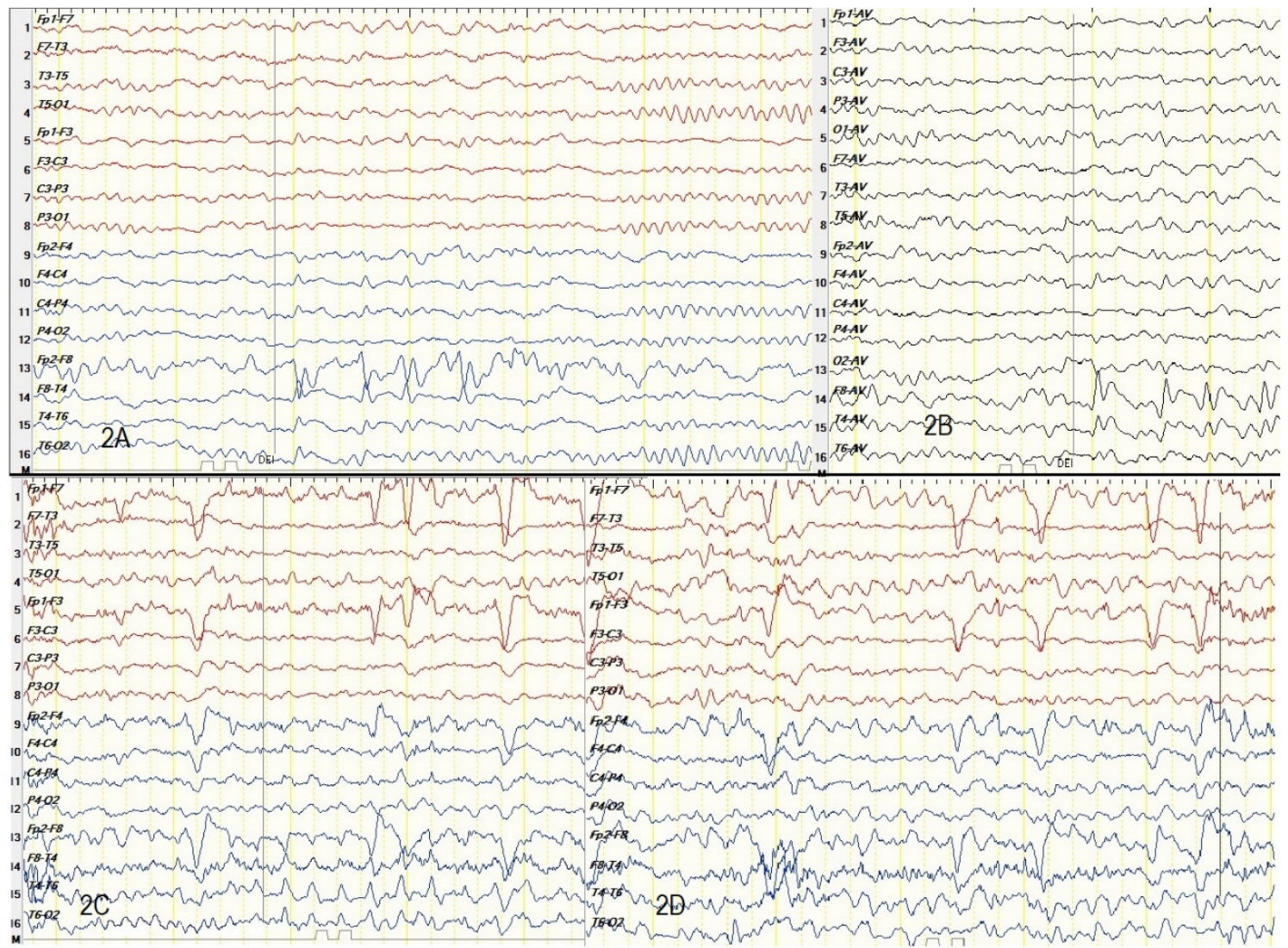

Figura 2. Descargas epileptiformes interictales: puntas secuenciales con mayor electronegatividad en F8 (2A montaje longitudinal; 2B montaje común promediado). Descarga epileptiforme ictal temporal derecha: actividad theta rítmica de morfología aguda con mayor electronegatividad en F8 (2C inicio de la crisis; 2D crisis en curso).

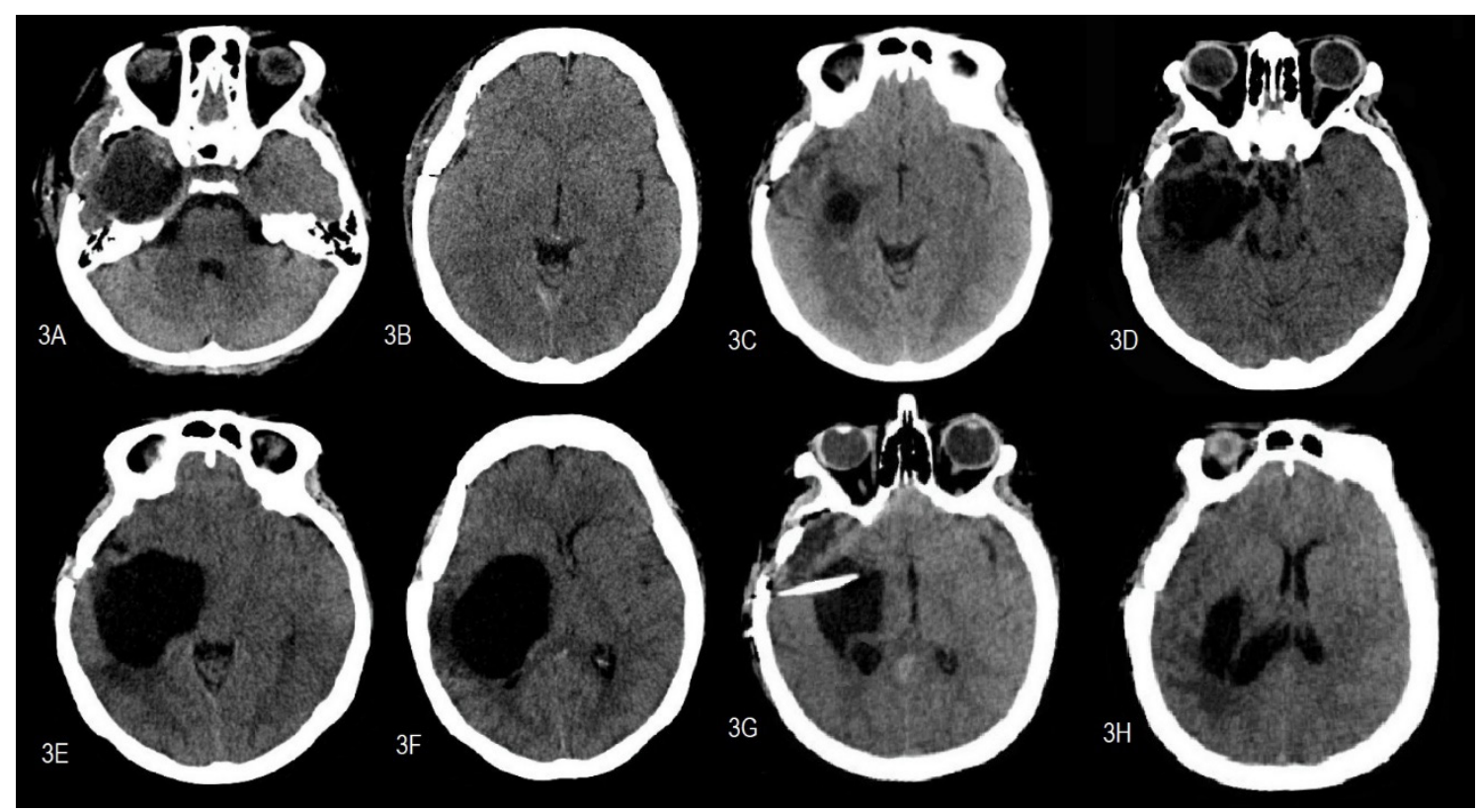

Figura 3. Tomografía cerebral post- lobectomía a las 48 horas (3A y 3B). Tomografía cerebral después de 7 meses de la cirugía evidencia pequeño quiste subcortical (3C). Tomografía cerebral después de 17 meses de la cirugía evidencia quiste gigante con efecto de masa (3D, 3E y 3F). Tomografía cerebral posterior a colocación de derivación cistoperitoneal evidencia reducción del volume del quiste $(3 \mathrm{G}$ y $3 \mathrm{H})$. 
cefalea se ha resuelto y ha mejorado significativamente de la hemiparesia izquierda.

\section{DISCUSIÓN}

La lobectomía temporal anterior es la técnica que ha probado, con nivel de evidencia IA, obtener libertad de crisis entre un $\sim 60$ a $73 \%$ de los pacientes con epilepsia focal farmacorresistente del lóbulo temporal. Es una técnica que además ha demostrado ser segura, aunque no libre de complicaciones. Un meta-análisis sobre estas complicaciones ha reportado la cuadrantanopsia homónima superior, los trastornos psiquiátricos (especialmente depresión y ansiedad) y el déficit cognitivo (principalmente el deterioro de memoria) como las complicaciones más frecuentes; con frecuencias estimadas entre 5 a $7 \%$. Es muy infrecuente la presentación de un quiste cerebral sintomático de instauración tardía como complicación de esta técnica.

En la literatura, solo se han reportado 2 casos similares al nuestro. Weaber et al, reportaron el caso de una paciente mujer de 24 años, con epilepsia temporal farmacorresistente por esclerosis mesial temporal derecha con edad de inicio de su epilepsia a los 20 años, quien después de 15 meses de la lobectomía temporal presentó cefalea, mareos y nauseas intermitentes; síntomas que se agravaron 12 meses después, asociándose a somnolencia y papiledema bilateral, constituyendo un síndrome de hipertensión endocraneana. En las neuroimágenes documentaron un quiste gigante de la fosa media derecha, que fue drenado mediante derivación cistoperitoneal. El procedimiento quirúrgico permitió la reducción del volumen del quiste y resolvió la afectación neurológica (5).

Bhaskara Rao et al., reportaron el caso de una mujer, también de 24 años con epilepsia que debutó a los 11 años (6). Ella presentaba epilepsia del lóbulo temporal derecho con resonancia de encéfalo negativa. Fue intervenida de una lobectomía temporal derecha con amigdalohipocampectomía, no describieron los hallazgos de la anatomopatología. Al cuarto mes de la cirugía las crisis focales recurrieron y se incrementaron gradualmente en los siguientes 5 meses, al décimo mes de la cirugía presentó cefalea, vómitos, papiledema y hemipaesia izquierda de instalación gradual. En la tomografía cerebral evidenciaron un gran quiste adyacente a la zona de resección que generaba efecto de masa y desplazaba la línea media; realizaron una nueva craneotomía, con una descompresión quística mediante una amplia escisión de la pared del quiste y de la gliosis periquística; el quiste contenía líquido cefalorraquídeo (LCR) e identificaron el ingreso del plexo coroideo del cuerno temporal del ventrículo lateral hacia el interior del quiste a través de un pequeño agujero comunicante. El plexo coroideo retenido dentro del quiste fue resecado. Dentro de la semana de la cirugía, la paciente mejoró de la cefalea, de la hemiparesia izquierda y quedó libre de crisis después de 8 meses de la cirugía (6).

Luego de la lobectomía temporal, el espacio del tejido resecado en la lobectomía es reemplazado por un determinado volumen de LCR, que está en libre comunicación con el LCR del sistema ventricular. Con el tiempo, el opérculo frontal y región posterior del lóbulo temporal circundantes se expanden, la cavidad con LCR en la zona de escisión se reduce a un volumen más pequeño que el de la región del lóbulo temporal resecado. Es muy inusual que el LCR quede loculado o atrapado en forma de un quiste y que se expanda hacia el parénquima cerebral vecino con efecto de masa. Respecto a la fisiopatología, tanto Weaber como Bhaskara Rao han propuesto que la obstrucción al flujo del LCR se generaría por un fenómeno de válvula; que podría ocurrir si el trígono o el cuerno temporal del ventrículo lateral fuesen constreñidos por una cicatriz o fibrosis del epéndimo, comprometiendo así la comunicación del LCR del lecho resecado con el cuerno temporal del ventrículo lateral. De esta forma el flujo obstruido del LCR ventricular sería forzado a organizar un quiste con expansión al parénquima vecino y entonces un flujo del LCR redireccionado se forzaría gradualmente al interior del quiste; en especial durante la fase inspiratoria de la respiración y durante la fase sistólica cardiaca. El plexo coroideo funcional del cuerno temporal ventricular podría estar expuesto al quiste, siendo entonces secuestrado dentro de una cavidad cerrada y favoreciendo aún más su llenado. Cuando el quiste adopte un determinado volumen, se comportará como una lesión expansiva sintomática $(5,6)$.

Debido a que estos quistes suelen estar revestidos por epéndimo y contener plexo coroideo, deben ser clasificados como neuroepiteliales y no como aracnoideos. Además, los quistes aracnoideos no suelen comportarse como procesos expansivos y no tienen comunicación con los ventrículos (7). En base a la experiencia sobre el manejo de los quistes aracnoideos; cuando un quiste intracerebral se comporta como una lesión expansiva sintomática, con hipertensión endocraneana o se asocia a déficit 
Quiste cerebral como complicación tardía inusual de lobectomía temporal en epilepsia farmacorresistente.

neurológico focal o a ocurrencia de crisis epilépticas.; requerirá de intervención quirúrgica. Habitualmente se han usado 3 técnicas quirúrgicas: craneotomía con escisión de la membrana del quiste, drenaje cistoperitoneal y fenestración del quiste; esta última técnica es la más utilizada pues evita la inserción permanente de un drenaje, pero el procedimiento implica una técnica quirúrgica más compleja y no siempre es exitosa (8). En nuestro caso se optó por la derivación cistoperitoneal debido a que en el contexto de la pandemia por COVID-19 se tenía tiempos acortados para cirugía por restricciones en el uso de sala de operaciones. Cabe mencionar, que además de las limitaciones quirúrgicas que impone el contexto de la pandemia, también limita el seguimiento ambulatorio adecuado de los pacientes post operados de epilepsia como se grafica en nuestro caso; pues la organización del quiste ya se insinuaba a los 7 meses después de la lobectomía, pero el seguimiento clínicotomográfico no se pudo realizar por las restricciones del contexto. La pandemia por COVID -19 ha tenido un impacto negativo en la respuesta de los servicios de salud en la atención de los pacientes con epilepsia (9).

En nuestra paciente, es evidente que el déficit neurológico focal subagudo se explica por el efecto expansivo del quiste. Respecto a la recurrencia de las crisis, además del efecto mecánico del proceso expansivo, se ha postulado que un incremento de niveles de aminoácidos excitatorios como Glutamato y Aspartato en el LCR contenido en el quiste favorecería la disminución del umbral epiléptico. El drenaje del líquido quístico excitatorio contribuiría a retomar el control de las crisis, como ocurrió en nuestra paciente (6).

En conclusión, la organización de un quiste cerebral sintomático, como complicación tardía de una lobectomía temporal, como parte del tratamiento de la epilepsia focal farmacorresistente, es muy infrecuente y hay que tenerla presente en un paciente expuesto a esta cirugía; que presentara tardíamente un déficit neurológico focal subagudo y recurrencia de crisis.

\section{Correspondencia}

Dr. Elliot Barreto Acevedo

Hospital Nacional Edgardo Rebagliati Martins

Av. Rebagliati 490 Jesús María

Teléfono: 511-265-4901 anexo: 3081. Celular: 51989963057.

Correo electrónico: elliotba@hotmail.com
Los autores no han recibido financiamiento de ninguna institución. El trabajo ha sido autofinanciado. Los autores no tienen conflicto de interés.

\section{REFERENCIAS BIBLIOGRÁFICAS}

1. McKhann G, Bourgeois B, Goodman R. Epilepsy Surgery: Indications, Approaches, and Results. Semin Neurol. 2002;22(3):269-78. doi: 10.1055/s2002-36653

2. Téllez-Zenteno J, Hernández L, Moien-Afshari F, Wiebe S. Surgical outcomes in lesional and non-lesional epilepsy: A systematic review and meta-analysis. Epilepsy Res. 2010;89(2-3):310-8. doi: 10.1016/j.eplepsyres.2010.02.007

3. Wiebe S, Blume W, Girvin J, Eliasziw M. A randomized, controlled trial of surgery for temporallobe epilepsy. N Engl J Med. 2001; 345(5):311-8. doi: 10.1056/NEJM2001080234505 01

4. Engel J, McDermott M, Wiebe S, Langfitt J, Stern J, Dewar S, et al. Early surgical therapy for drug-resistant temporal lobe epilepsy. JAMA. 2012; 307(9):922-30.

5. Weaver JP, Phillips C, Horowitz SL, Benjamin S. Middle fossa cyst presenting as a delayed complication of temporal lobectomy-case report. Neurosurgery. 1996; 38(5): 1047-50; disussion 1050-1. doi: 10.1097/00006123-199605000-00040

6. Bhaskara Rao M, Radhakrishnan K, Radhakrishnan V, Gupta K. Expanding cyst following temporal lobectomy: an unusual complication of epilepsy surgery. Clin Neurol Neurosurg. 1999;101(2):1414. doi: 10.1016/s0303-8467(99)00021-9

7. Go KG, Hew JM, Kamman RL, Molenaar WM, Pruim J, Blaauw EH. Cystic lesions of the brain. A classification based on pathogenesis, with consideration of histological and radiological features. Eur J Radiol. 1993;17(2):69-84. doi: 10.1016/0720-048x(93)90038-o

8. Padilla G, Jallo G. Arachnoid cyst: case series and review of the literature. Neurosurg Focus. 2007; 22(2):E7. doi: 10.3171/foc.2007.22.2.7

9. Adan G, Mitchell J, Marson T. Epilepsy care in the COVID-19 era. Clin Med (Lond). 2020;20(4):e104-e106. doi: 10.7861/ clinmed.2020-0207

Recibido: 21/01/2021

Aceptado: 03/05/2021 